05

\title{
Применение новой модели остаточной намагниченности железа для расчета поворотного магнита ускорителя
}

\author{
() М.Э. Рояк ${ }^{1}$, И.М. Ступаков ${ }^{1}$, Н.С. Кондратьева ${ }^{1}$, \\ Н.А. Винокуров 2 ॠ , О.А. Шевченко ${ }^{2}$, \\ С.С. Середняков ${ }^{2}$, Я.И. Горбачёв ${ }^{2}$ \\ ${ }^{1}$ Новосибирский государственный технический университет \\ ${ }^{2}$ Институт ядерной физики им. Г.И. Будкера СО РАН, Новосибирск \\ I E-mail: vinokurov@inp.nsk.su
}

Поступило в Редакцию 27 декабря 2016 г.

Учет остаточной намагниченности железа необходим при расчете магнитного поля элементов электронно-оптических каналов ускорителей заряженных частиц, так как вклад остаточной намагниченности в поле в рабочем зазоре может быть порядка mT. Кроме того, величина и знак этого вклада зависят от процесса намагничивания. Ранее авторами была предложена модель остаточной намагниченности железа, использующая небольшое число сортов магнитных доменов. Здесь описан алгоритм расчета магнитного поля с применением этой модели. Работоспособность алгоритма продемонстрирована для случая простейшего электромагнита с $O$-образным магнитопроводом. Сравнение с результатами измерений показало удовлетворительную точность учета остаточной намагниченности.

DOI: 10.21883/PJTF.2017.20.45147.16647

В большинстве существующих программных комплексов для расчета магнитных полей фрагментов ускорителей используется подход, основанный на использовании зависимости магнитной проницаемости среды от поля, рассчитанной по главной кривой намагничивания. Этот подход никак не учитывает гистерезис, что часто приводит к расхождению рассчитанных характеристик магнита с экспериментальными. Основная цель настоящей публикации - разработка эффективных методов численного моделирования магнитных полей в магнитах с ферромагнитным магнитопроводом, позволяющих учитывать остаточную 
намагниченность ферромагнетиков, зависящую от „истории“ изменения возбуждающих поле токов.

Будем считать, что вся область моделирования состоит из двух подобластей: подобласти $\Omega_{H}$, в которой отсутствуют сторонние токи и содержатся все магнитные материалы, и подобласти $\Omega_{A}$, в которой нет магнитных материалов и есть сторонние токи. Используем математическую модель на основе полного и неполного скалярных потенциалов [1].

Представим напряженность поля $\mathbf{H}$ в подобласти $\Omega_{H}$ в виде градиента полного потенциала: $\mathbf{H}=-\nabla u$. Тогда индукция определяется формулой $\mathbf{B}=\mu_{0}(-\nabla u+\mathbf{M})$, где $\mu_{0}-$ магнитная проницаемость вакуума, М - намагниченность среды, которая может зависеть от напряженности поля, времени и предыстории намагниченности, а из условия $\nabla \mathbf{B}=0$ получаем уравнение для нахождения полного скалярного потенциала $u$ в подобласти $\Omega_{H}$

$$
\Delta u=\nabla \mathbf{M}
$$

Построим для уравнения (1) вариационную постановку. Запишем это уравнение в слабой форме, домножив обе его части на некоторую пробную функцию $v$ из пространства $H^{1}$ и проинтегрировав их по $\Omega_{H}$. Тогда, используя интегрирование по частям и то, что $\frac{1}{\mu_{0}} \mathbf{B}=-\nabla u+\mathbf{M}$, получим

$$
-\frac{1}{\mu_{0}} \oint_{\partial \Omega_{H}} \mathbf{B} \cdot \mathbf{n} v d \Gamma=\int_{\Omega_{H}} \nabla u \cdot \nabla v d \Omega-\int_{\Omega_{H}} \mathbf{M} \cdot \nabla v d \Omega .
$$

В подобласти $\Omega_{A}$ будем искать $\mathbf{H}$ в виде $-\nabla u+\mathbf{H}_{e x t}$, где $u-$ неполный потенциал, $\mathbf{H}_{e x t}-$ поле токов в однородном пространстве, удовлетворяющее уравнению $\nabla \cdot \mathbf{H}_{e x t}=0$. Тогда для нахождения неполного потенциала $u$ в подобласти $\Omega_{A}$ получаем уравнение $\Delta u=0$. Аналогично выводу (2), записывая вариационную постановку в подобласти $\Omega_{A}$, получим

$$
-\frac{1}{\mu_{0}} \oint_{\partial \Omega_{A}} \mathbf{B} \cdot \mathbf{n} v d \Gamma=\int_{\Omega_{A}} \nabla u \cdot \nabla v d \Omega-\oint_{\partial \Omega_{A}} \mathbf{H}_{e x t} \cdot \mathbf{n} v d \Gamma .
$$

На границе между подобластями необходимо потребовать непрерывности нормальной компоненты вектора В и касательной компоненты

Письма в ЖТФ, 2017, том 43, вып. 20 
вектора Н. Первое условие обеспечивается, если сложить уравнения (2) и (3) и учесть, что подынтегральные выражения их левых частей различаются на границе подобластей только знаком нормали:

$$
\int_{\Omega} \nabla u \cdot \nabla v d \Omega=\int_{\Omega_{H}} \mathbf{M} \cdot \nabla v d \Omega+\oint_{\partial \Omega_{A}} \mathbf{H}_{e x t} \cdot \mathbf{n} v d \Gamma .
$$

Для выполнения второго условия потенциал $u$ должен быть полным в подобласти $\Omega_{H}$ и неполным в подобласти $\Omega_{A}$, т.е. функция должна иметь разрыв на границе между подобластями, и касательная компонента градиента этого разрыва должна быть равна касательной компоненте вектора $\mathbf{H}_{\text {ext }}$. Это можно обеспечить специальным выбором базисных функций для представления $u$.

Для построения конечно-элементной дискретизации представим потенциал $u$ в виде разложения по базису $u=\sum_{j} q_{j} \varphi_{j}$ и выберем пробные функции из того же конечно-мерного пространства. Тогда для пробной функции $\varphi_{i}$ уравнение (4) примет вид

$$
\sum_{j} q_{j} \int_{\Omega} \nabla \varphi_{i} \cdot \nabla \varphi_{j} d \Omega=\int_{\Omega_{H}} \mathbf{M} \cdot \nabla \varphi_{i} d \Omega+\oint_{\partial \Omega_{A}} \mathbf{H}_{e x t} \cdot \mathbf{n} \varphi_{i} d \Gamma .
$$

Будем считать, что на каждом конечном элементе $\Omega_{k}$ намагниченность $\mathbf{M}^{\Omega_{k}}$ постоянна и зависит от среднего на элементе значения напряженности. Введем обозначения $A_{i j}=\int_{\Omega} \nabla \varphi_{i} \times$ $\times \nabla \varphi_{j} d \Omega, \mathbf{B}_{i}^{k}=\int_{\Omega_{k}} \nabla \varphi_{i} d \Omega, f_{i}=\oint_{\partial \Omega_{A}} \mathbf{H}_{e x t} \cdot \mathbf{n} \varphi_{i} d \Gamma$, тогда $\int_{\Omega_{H}} \mathbf{M} \cdot \nabla \varphi_{i} d \Omega \approx$ $\approx \sum_{k} \mathbf{M}^{\Omega_{k}}\left(\mathbf{H}^{k}\right) \cdot \mathbf{B}_{i}^{k}$, где $\mathbf{H}^{k}=-\frac{1}{\left|\Omega_{k}\right|} \sum_{i} q_{i} \mathbf{B}_{i}^{k}$, а систему уравнений (5) можно записать в матричном виде

$$
\mathbf{A q}=\mathbf{f}+\sum_{k} \mathbf{F}^{\Omega_{k}}(\mathbf{q})
$$

где матрица $\mathbf{A}$ состоит из компонент $A_{i j}$, вектор $\mathbf{q}-$ из компонент $q_{i}$, вектор $\mathbf{f}-$ из компонент $f_{i}$, а вектор $\mathbf{F}^{\Omega_{k}}-$ из компонент

$$
F_{i}^{\Omega_{k}}(\mathbf{q})=\mathbf{M}^{\Omega_{k}}(\mathbf{H}) \cdot \mathbf{B}_{i}^{k} .
$$

Письма в ЖТФ, 2017, том 43, вып. 20 
Обратим внимание на то, что система уравнений (6) является нелинейной. Поскольку ее решение методом простой итерации, как показали вычислительные эксперименты, оказывается чрезвычайно неэффективным, построим итерационный процесс с линеаризацией этой системы в окрестности некоторого начального решения $\mathbf{q}^{0}$ :

$$
F_{i}^{\Omega_{k}}(\mathbf{q})=F_{i}^{\Omega_{k}}\left(\mathbf{q}^{0}\right)+\sum_{j} \frac{\partial F_{i}^{\Omega_{k}}}{\partial q_{j}}\left(q_{j}-q_{j}^{0}\right),
$$

где с учетом (7)

$$
\frac{\partial F_{i}^{\Omega_{k}}}{\partial q_{j}}=\sum_{l=1}^{3} B_{i l}^{k} \sum_{m=1}^{3} \frac{\partial \mathbf{M}_{l}^{\Omega_{k}}}{\partial \mathbf{H}_{m}} \frac{\partial \mathbf{H}_{m}}{\partial q_{j}}=-\frac{1}{\left|\Omega_{k}\right|} \sum_{l=1}^{3} \sum_{m=1}^{3} \frac{\partial \mathbf{M}_{l}^{\Omega_{k}}}{\partial \mathbf{H}_{m}} B_{i l}^{k} B_{j m}^{k} .
$$

Выражение (8) фактически определяет вклады в матрицу и правую часть от конечного элемента для метода Ньютона (см. [1]).

Используем для описания зависимости $\mathbf{M}^{\Omega_{k}}(\mathbf{H})$ на конечном элементе одну из скалярных макромоделей вида $M(H)$, рассмотренных в [2]. Для перехода от скалярной модели к изотропной векторной можно использовать усреднение по направлениям [3], т.е.

$$
\mathbf{M}(\mathbf{H})=\frac{3}{4 \pi} \int_{S} \mathbf{r} M(\mathbf{H} \cdot \mathbf{r}) d S,
$$

где $S$ - сфера единичного радиуса. Вычислим интеграл в (9) численно. Обозначим через $N$ число точек используемой квадратуры, через $\mathbf{r}_{n}$ сами точки, а через $w_{n}-$ соответствующие им веса с учетом коэффициента нормировки. Тогда векторная макромодель будет иметь вид $\mathbf{M}(\mathbf{H}) \approx \sum_{n=1}^{N} w_{n} \mathbf{r}_{n} M\left(\mathbf{H} \cdot \mathbf{r}_{n}\right)$, и вместо (7) получим

$$
F_{i}^{\Omega_{k}}(q)=\sum_{n=1}^{N} w_{n} M\left(\mathbf{H} \cdot \mathbf{r}_{n}\right)\left(\mathbf{B}_{i}^{k} \cdot \mathbf{r}_{n}\right) .
$$

Обозначим через $\mathbf{r}_{n, l}$ компоненту $l$ вектора $\mathbf{r}_{n}$. Тогда производную компоненты вектора намагниченности по компоненте вектора напряженности можно вычислить по формуле

$$
\frac{\partial \mathbf{M}_{l}}{\partial \mathbf{H}_{m}}=\sum_{n=1}^{N} w_{n} \mathbf{r}_{n, l} \mathbf{r}_{n, m} M^{\prime}\left(\mathbf{H} \cdot \mathbf{r}_{n}\right)
$$

Письма в ЖТФ, 2017, том 43, вып. 20 


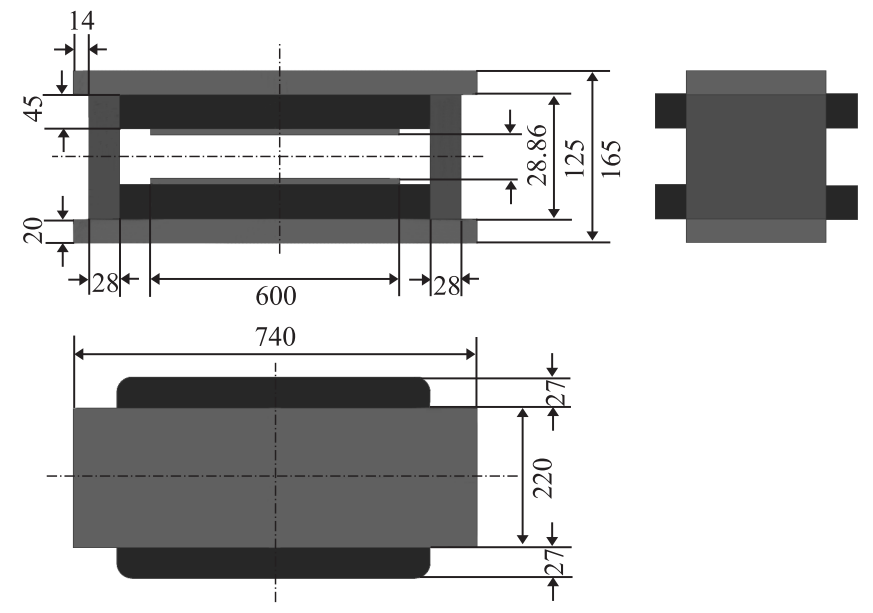

Рис. 1. Поворотный магнит.

Подставив полученное выражение в (8), получим

$$
\frac{\partial F_{i}^{\Omega_{k}}}{\partial q_{j}}=-\frac{1}{\left|\Omega_{k}\right|} \sum_{n=1}^{N} w_{n} M^{\prime}\left(\mathbf{H} \cdot \mathbf{r}_{n}\right)\left(\mathbf{B}_{i}^{k} \cdot \mathbf{r}_{n}\right)\left(\mathbf{B}_{j}^{k} \cdot \mathbf{r}_{n}\right) .
$$

Таким образом, реализация учета гистерезиса требует хранения на каждом конечном элементе $N$ комплектов параметров истории макромодели гистерезиса, т. е., например, для модели Винокурова, описанной в [2], $-2 N$ вещестнных чисел.

Для проверки точности нового алгоритма, учитывающего остаточную намагниченность, были проведены магнитные измерения поля в зазоре типичного поворотного магнита (рис. 1) при циклическом изменении тока в обмотке. Чтобы усилить гистерезисные явления, боковые стенки $O$-образного магнитопровода были изготовлены из конструкционной стали 3 с относительно высоким содержанием углерода. Именно для этой стали изучались модели гистерезиса в работе [2]. На обмотку подавался ток с пилообразной временно́й зависимостью. Все измерения проводились из предварительно размагниченного состояния при симметричном (биполярном) и несимметричном (уни- 


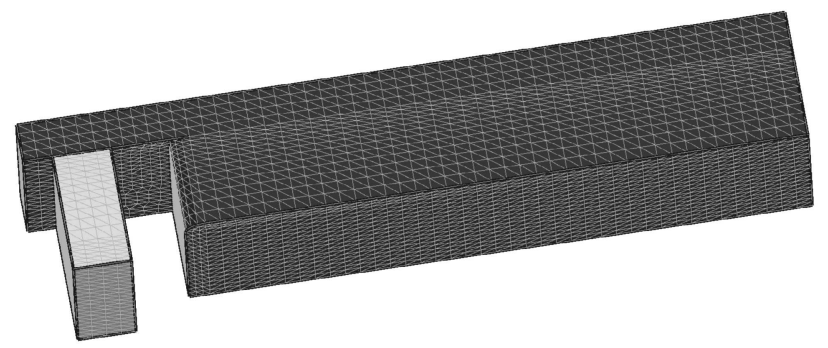

Рис. 2. Сетка на поверхности стали.

полярном) изменении тока $I$ в обмотке. Измерения датчиком Холла проводились в двух точках: первая находится в центре магнита, а вторая сдвинута на $25 \mathrm{~cm}$ вдоль длинной стороны полюса.

Для численного моделирования была задана восьмая часть магнита, построена сетка, изображенная на рис. 2, и использована следующая скалярная модель гистерезиса [2]:

$$
M(H)=\chi H+\frac{1}{2}\left(M\left(H_{e f f_{1}}\right)+M\left(H_{e f f_{2}}\right)\right),
$$

где $M\left(H_{e f f}\right)=M_{\max }\left(1-1 / \sqrt{1+2 \chi_{0}\left|H_{e f f}\right| / M_{\max }}\right) \operatorname{sgn}\left(H_{e f f}\right), H_{e f f_{i}}$ находится как решение дифференциального уравнения $d H_{\text {eff }}=$ $=\vartheta\left[\left|H \cos \theta_{i}-H_{e f f_{i}}\right|-H_{c_{i}}\right] \cos \theta_{i} d H, \vartheta-$ ступенчатая функция Хевисайда, $\theta_{1}=\pi / 6, \theta_{2}=\pi / 3$. Отличие от описанной в работе [2] модели состоит только в том, что в формуле (11) присутствует дополнительное слагаемое $\chi H$. Для стали 3 параметры векторной модели были подобраны по экспериментальным данным, приведенным в работе [2]: $M_{\max }=793 \mathrm{G}, \chi=0, \chi_{0}=1295, H_{c_{1}}=1.43$ Ое и $H_{c_{2}}=0.39$ Ое. Полюс магнитопровода изготовлен из малоуглеродистой стали, по химическому составу и магнитным свойствам близкой к чистому железу. Однако численное моделирование было осложнено тем, что образцов этой стали не осталось, и поэтому определять параметры модели гистерезиса для расчета пришлось по трехмерному моделированию. Для подбора параметров модели было выбрано симметричное изменение тока. Таким образом были получены параметры для этой стали $M_{\max }=472 \mathrm{G}$, $\chi=397, \chi_{0}=4802, H_{c_{1}}=1.48$ Ое и $H_{c_{2}}=0.23$ Ое. Результаты модели-

3 Письма в ЖТФ, 2017, том 43, вып. 20 
\ Measurements

- Calculations a

$b$
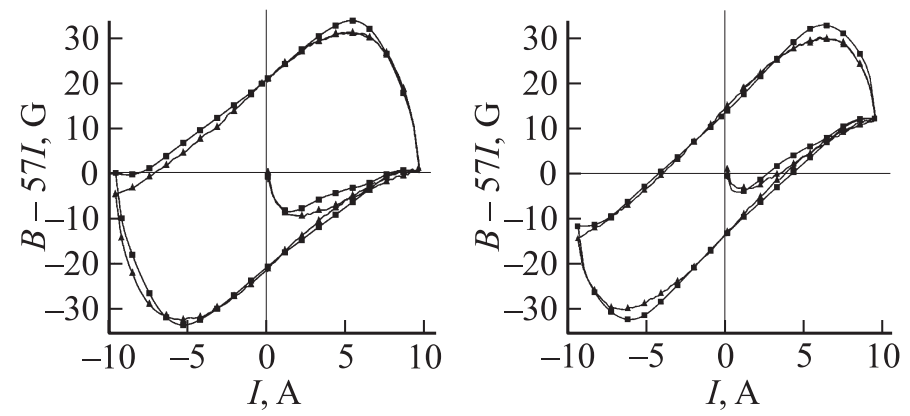

C

$d$
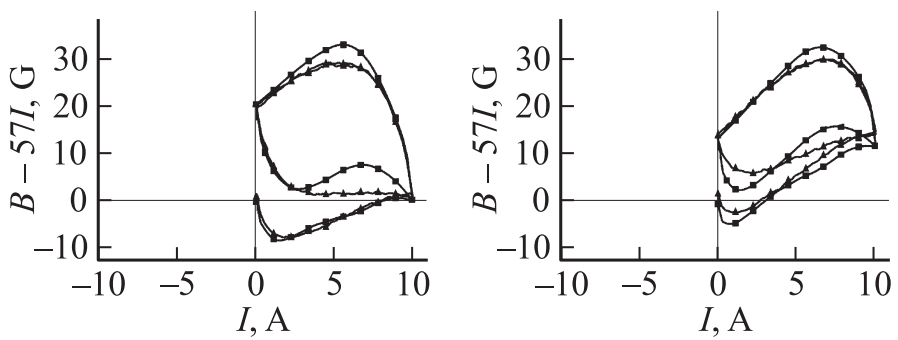

Рис. 3. Измеренные (треугольники) и рассчитанные (квадраты) зависимости индукции $B$ в двух точках ( $a$ и $c-$ в центре, $b$ и $d-$ на краю) зазора электромагнита от тока $I$ в его катушках. По оси ординат отложено поле с вычтенной линейной частью, т. е. величина $B(\mathrm{G})-57 I(\mathrm{~A})$.

рования вместе с данными экспериментов представлены на рис 3. Для того чтобы отличия эксперимента от модели были видны, на рисунках показаны графики функции $B-57 I$. Измерения и результаты представлены в двух точках. Первая находится в центре полюса (рис. 3, $a, c$ ), а вторая - в $25 \mathrm{~cm}$ от центра (рис. $3, b, d$ ). Измерения и расчеты проводились при двух способах изменения тока: симметричном, когда ток менялся циклически от -8 до $+8 \mathrm{~A}$ (рис. $3, a, b)$, и несимметричном, когда ток менялся циклически от 0 до $8 \mathrm{~A}$ (рис. $3, c, d$ ). 
Из представленных результатов видно, что симметричный цикл, по которому подбирались параметры модели, описывается несколько точнее, но и для несимметричного цикла отличия моделирования от эксперимента не превышают 5-6 G. Заметим, что частично эти отличия могут объясняться вихревыми токами, которые не учитываются в описанной в настоящей работе модели. Моделирование вихревых токов, проведенное с использованием не учитывающего гистерезис подхода, описанного в [4], показало, что вихревые токи вносят вклад более $2 \mathrm{G}$, что подтверждается и экспериментально (при использовании другой скорости подъема тока). Остальные отличия могут объясняться как недостаточно хорошо подобранными параметрами модели гистерезиса, так и неполным размагничиванием перед началом циклов. Следует отметить, что такая ситуация типична для магнитов, используемых в ускорительной технике, так как обычно магнитопроводы электромагнитов конструируются так, чтобы индукция в них не достигала индукции насыщения. Заметим, что даже описанная выше довольно грубая аппроксимация гистерезисных явлений обеспечивает точность расчета, в несколько раз лучшую, чем обычно используемые расчеты по главной кривой намагничивания, не учитывающие остаточной намагниченности (порядок ошибок расчетов без учета остаточной намагниченности высота петель гистерезиса, показанных на рис. 3). Кроме того, точность знания параметров петель гистерезиса материала магнитопровода тоже невелика, так как эти параметры зависят от технологии изготовления, в частности от температуры отжига, которая может быть различной у разных образцов того же материала и даже в разных областях одной и той же детали. Полученные результаты сравнения данных эксперимента и численного моделирования показывают несомненную перспективность рассматриваемого подхода к учету гистерезиса в численном моделировании. Однако для построения эффективных процедур численного моделирования необходимы дальнейшие исследования, в частности требуется внести в математическую модель возможность учета вихревых токов, возникающих в полюсе магнита при изменении тока в обмотках; также нужно провести эксперименты с магнитом, для всех материалов которого можно отдельно оценить параметры модели гистерезиса.

Работа выполнена при поддержке Российского фонда фундаментальных исследований (грант № 15-02-07776). Модификация электромагнита и измерения магнитного поля в нем выполнены за счет гранта Российского научного фонда (проект № 14-50-00080).

$3^{*}$ Письма в ЖТФ, 2017, том 43, вып. 20 


\section{Список литературы}

[1] Соловейчик Ю.Г., Рояк М.Э., Персова М.Г. Метод конечных элементов для скалярных и векторных задач. Новосибирск: НГТУ, 2007. 869 с.

[2] Винокуров Н.А., Шевченко О.А., Середняков С.С., Щеглов М.А., Рояк М.Э., Ступаков И.М., Кондратьева Н.С. // Письма в ЖТФ. 2016. Т. 42. В. 13. C. $96-103$.

[3] Mayergoyz I.D. Mathematical models of hysteresis and their applications. Academic Press, 2003. 498 p.

[4] Royak M.E., Stupakov I.M., Kondratyeva N.S. // Actual problems of electronic instrument engineering (APEIE-2016). Proc. of the 13th Int. Conf. Novosibirsk: NSTU, 2016. V. 1. Pt 2. P. 330-335. 